\title{
Expectativas, necesidades y tendencias de la formación en educación superior en Colombia en pregrado y posgrado: entre la deserción-perfil y vocación profesional $^{*}$
}

\author{
Jahir A. Gutiérrez O.** \\ Viviana Mondragón M.** $^{* \star}$ \\ Laura C. Santacruz M. ${ }^{* * * *}$
}

Fecha de recibido: 18 de marzo de 2018

Fecha de aprobado: 25 de octubre de 2018

Para citar: Gutiérrez, J.A, Mondragón, V., \& Santacruz, L. (2019). Expectativas, necesidades y tendencias de la formación en educación superior en Colombia en pregrado y posgrado: entre la deserción-perfil y vocación profesional.

Universidad E Empresa, 21(37), 313-345.

Doi: http://dx.doi.org/10.12804/revistas.urosario.edu.co/empresa/a.6619

* Este artículo es producto del proyecto de investigación: "Expectativas-necesidades y tendencias de la formación en educación superior en Colombia en pregrado y posgrado: entre la deserción, perfil y vocación profesional". Ejecutado por el Grupo de Investigación en Gestión Empresarial de la Facultad de Administración de Empresas y Ciencias Económicas de la Universidad CES. Aprobado por la Dirección de Gestión del Conocimiento de la Universidad CES. Código interno INV032014001. Fecha de inicio: Julio 01 de 2014. Fecha de Terminación: Julio 01 de 2015. Artículo que hace parte del contenido de la Maestría en Gestión Educativa a ofertarse en la Universidad CES, Medellín, Colombia.

** Coordinador y líder del Grupo de Investigación en Gestión Empresarial de la Universidad CES, avalado por la Dirección de Gestión del Conocimiento. Economista industrial de la Universidad de Medellín, Colombia. Magíster en Desarrollo con énfasis en lo Regional y lo Local de la Universidad Pontificia Bolivariana, sede Medellín. PhD. en Administración Pública de la Atlantic International University, Honolulu, USA. Dirección Profesional: Calle 10A\# 22-04. Bloque C. Oficina 1. Teléfono: 444 0555. Ext. 1411.

*** Jefe de posgrados Facultad de Ciencias Administrativas y Económicas en la Universidad CES. Ingeniera industrial de la Universidad Nacional de Colombia, con experiencia en el sector educativo y productivo. Magíster profesional en Dirección y Gestión de Recursos Humanos del CEF. Magíster en Administración con énfasis en estrategia del TEC de Monterrey. Dirección: Calle 10 A \# 22- 04. Bloque C. Oficina 2. Tel: 4440555. Ext. 1429.

**** Estudiante de Administración de Empresas de la Universidad CES, séptimo semestre. Integrante del Grupo de Investigación en Gestión Empresarial de la Universidad CES. Correo electrónico: caroliinasantacruz@gmail.com Celular: 3205351435 


\section{Resumen}

El escrito evalúa las condiciones que presenta la educación de pregrado y posgrado para acercar los conceptos de economía y mercado a la educación superior en Colombia, la cual ha sido impartida bajo la óptica conductual y funcionalista, sin albergar espacio para dicho acercamiento. El análisis multicriterio, sirve de fundamento metodológico para plantear la evaluación, mientras que los temas a tratar responden al estado del arte en materia de evaluación de la educación superior. Seguido, se presentan los impactos y resultados obtenidos de la evaluación internacional en ambos grados educativos, y finalmente, el espacio que tiene el pregrado y el posgrado para medirse en términos de la economía y el mercado de la educación en Colombia, ya que la educación superior tiene dificultades para mostrarse positiva.

Palabras clave: educación superior, perfil académico y profesional, deserción académica y profesional, economía de la educación superior, impactos y resultados de la educación superior en Colombia.

\section{Expectations, Needs and Trends of Higher Education in Colombia in Pre-grad and Post-grad: Between Desertion Profile and Profetional Vocation Abstract}

Brief evaluates the conditions presented by undergraduate and postgraduate education to bring the concepts of economy and market to higher education in Colombia, which has been given under the optical behavioral and functionalist, without hosting space for purposes realesdicho approach. The Multicriteria analysis, serves as methodological basis for evaluation, while the topics correspond to the State of the art in evaluation of higher education. Followed, are the impacts and results of the international evaluation in both educational degrees, and finally, space that has the undergraduate and the graduate to be measured in terms of the economy and the market of education in Colombia, since the higher education has difficult to show positive.

Keywords: Higher education, profile academic and professional, desertion academic and professional, economy of higher education, impacts and results of the superior education in Colombia.

\section{Expectativas, necessidades e tendências da formação em educação superior na Colômbia em graduação e pós-graduação: entre a deserção, perfil e vocação profissional \\ Resumo}

Breve avalia as condições apresentadas pelos cursos de graduação e pós-graduação para trazer os conceitos de economia e mercado de ensino superior na Colômbia, que tem sido dado sob a ótica comportamental e funcionalista, sem Hospedagem de espaço para abordagem de realesdicho fins. A análise Multicritério, serve como base metodológica para a avaliação, enquanto os tópicos correspondem ao estado da arte em avaliação do ensino superior. Seguido, são os impactos e resultados da avaliação internacional em graus de ensino e finalmente, espaço que tem os cursos de graduação e pós-graduação a ser medido em termos de economia e o mercado da educação na Colômbia, desde que o ensino superior tem difícil mostrar positivo.

Palavras-chave: educação Superior, Perfil Acadêmico e Profissional, Deserção Acadêmica e Profissional, Economia da Educação Superior, Impactos e Resultados da Educação Superior na Colômbia. 


\section{Introducción}

Es importante atender la deserción como punto referente de una serie de elementos mucho más disientes. La combinación entre perfil y vocación debe elevar la medición estrictamente apegada al grueso de estudiantes que abandonan el estudio, en aras de fortalecer los mecanismos de interacción u observancia, que podrían ampliar aún más el debate sujeto a la dejación de los estudios. El trabajo sobre el perfil académico debe entregar una especie de "plancha académica" sobre la cual el estudiante se entienda en el marco de la senda de la formación profesional, en tanto que, en materia de vocación, esta última atiende los campos en que efectivamente el profesional es capaz de poner a disposición su carrera.

La base de la educación superior tecnológica y universitaria en el país está expuesta a factores que intentan establecer formas diferentes de entender la formación; la estructura académica de la formación superior debe responder precisamente a distintos frentes, entre los que se cuenta la oferta de programas. En dicho sentido, ha de considerarse el impacto que puede crear en el medio el cambio o reposición de carreras tradicionales o si necesariamente la institucionalidad universitaria (tecnológica-universidad), debe alistarse para cambios más profundos respecto al ofrecimiento de programas cuya concepción no necesariamente haya cambiado, así como revisar las alteridades que presenta el mercado en cuanto al impacto real sobre la oferta académica.

El análisis multicriterio permite analizar problemas o temas desde la perspectiva cuantitativa y cualitativa, y acoge los fundamentos teóricos, como los ofrecidos en materia de cifras, datos y números. A diferencia de otras metodologías, toma una de ellas o ambas para construir criterios hacia el otro frente, es decir que, en ausencia de alguna, exige llegar a los lineamientos del elemento faltante; además, permite trabajar a través de la postulación de modelos, las pruebas de ajuste por medio de la estadística y de la observación de datos por intermedio del análisis data panel, al igual que accede a la vinculación de cartografía, esquemas y mapas con los cuales poder orientar el problema desde diferentes tópicos, sin perder de vista el tema central de análisis desde el principio y hasta el final. 
La posibilidad de habilitar de manera permanente mecanismos con los cuales poder trabajar en la materia es una tarea que convoca a las instituciones de educación superior (IES). La orientación, el perfil y la vocación son elementos contundentes para revertir los análisis que hasta ahora han puesto a la deserción un cumulo de cantidades y tareas para atajarla. Es el paso activo de la educación el que debe ahondar en los campos sobre los cuales pueda evidenciarse claramente que el tema en sí obedece solo a una parte de lo que significa ofrecer plenamente servicios educativos en todos los escenarios, en los que la universidad debe tener una actuación más profunda de lo logrado hasta ahora, y que pone a la deserción, en el escenario no esperado, pero tampoco se extiende el análisis.

El artículo comporta las siguientes temáticas de revisión para su consideración: 1. Contexto de la formación en educación superior frente a la deserción académica y la expectativa profesional; 2. Perspectiva de la educación superior en pregrado y posgrado en Colombia frente a la deserción profesional y 3. La economía de la educación superior en la formación de pregrado y posgrado en Colombia. En las IES deben pasarse a la evaluación de cifras concretas en materia de los impactos y resultados que trae la formación profesional en el tiempo, y ello conlleva a comprometerse más en cuanto a los perfiles profesionales, la remuneración y los grados de respuesta de la sociedad, dado que para las IES este será el nuevo marco de trabajo de la formación profesional.

\section{Contexto de la formación en educación superior frente a la deserción académica y la expectativa profesional}

La elaboración de los análisis, estudios e investigaciones generadas en materia de oferta académica y educativa a escala superior han girado en torno a la calidad, la cobertura y la deserción, ganando esta dos últimas la partida; el incremento de la población estudiantil en términos nominales no ha terminado por opacar los datos reales cuando se trata de evidenciar las falencias mostradas en la educación superior. La deserción como primera guía de análisis solo ha terminado por afianzar los diagnósticos elaborados del tema; así mismo, la cobertura se ha convertido en un afán por capturar recursos públicos 
y/o privados destinados al fomento de la educación. En ambos contextos, la calidad es poco advertida o considerada en esencia para poder revertir los problemas presentados.

[...] El concepto de deserción -tomado del lenguaje militar- establece una relación directa con el acto voluntario de dejar un determinado lugar, institución o situación [...]. En el caso de la deserción escolar, entendida como el acto de retiro escolar-transitorio o prolongado en el tiempo-, en su mayoría se debe a situaciones dadas dentro de la institución escolar (Ricoachury \& Lara, 1984, citados en Hincapié et al., 2012, p. 18).

Es imperante para las instituciones de educación superior y las universidades tener un amplio bagaje en cuanto a lo implica la triada deserción-perfil-vocación, en cuanto a lo que implica para esta orientar trabajo antes, durante y después del periodo de graduación de los estudiantes. El papel que cumple la institucionalidad no solo se remite al otorgamiento de títulos, sino que deben realizarse esfuerzos encaminados a la observancia, registro y postulación de iniciativas que permitan mejorar las condiciones de la carrera o profesión antes del inicio de la misma, en medio del proceso de formación y luego con mucha más lógica en la propia etapa de formación, actividad que debería estar en cabeza de las unidades de gestión estudiantil y de egresados en sí.

Si nos centramos en el ámbito de la educación universitaria, las relaciones educación-empleo se vuelven aún más complejas; esto se debe, principalmente, al amplio abanico de carreras y especializaciones ofertadas por la Universidad que a veces no se identifican con los contenidos de los empleos de la economía. Además, en muchas ocasiones los empleadores desconocen qué titulaciones se ofertan y qué papel podrían jugar en la empresa. Otras veces, aun conociendo los títulos y contenidos de las carreras, los empleadores se quejan de que la universidad sólo transmite conocimientos técnicos e intelectuales, pero no contribuye a desarrollar habilidades que se consideran hoy día imprescindibles para el desempeño de trabajos cualificados, los cuales en una economía se suelen asignar (Salas, 2004, p. 260).

El incremento de la oferta académica en los niveles de pregrado y posgrado no implica un cambio de dirección o estabilización del sistema académico y educativo de las instituciones de educación superior. Es evidente que el aumento de la población incide en el incremento de la población interesada en formarse en la educación superior, más esto no 
implica que estas encuentren como punto de balance que el incremento en la población activa estudiantil o que la decisión que esta tome para con las respondientes carreras sea un punto de referencia amplio para cohonestar la perspectiva de una carrera con la posible deserción, perfil o vocación que dicho contexto podría tamizar realmente.

No se conoce sino imperfectamente el impacto de la educación y la capacitación sobre el desempeño en el mercado del trabajo. Es costumbre utilizar los salarios como indicador y así se hará en la siguiente serie de análisis. Se parte del supuesto de que un mejor desempeño en el mercado de trabajo será recompensado con remuneraciones más altas y que las diferencias de remuneración por niveles educativos constituyen una medida de los incentivos financieros que se ofrecen en un país determinado para efectuar mayores inversiones en educación (OCDE, 1997). Sin embargo, estas hipótesis presentan evidentes limitaciones. Las diferencias de ingresos laborales pueden reflejar también diferencias en la oferta de programas educativos de distintos niveles y en el acceso a ellos (Carlson, 2002, p. 133).

La educación superior a escala tecnológica y universitaria en el país, tanto al nivel de pregrado como de posgrados, exige una revisión permanente por parte de las instituciones de educación adscritas a dicho campo, ya que no basta solo obtener datos y estudios, sino que se debe establecer un criterio más argumentativo y aprehensivo frente a lo que implica revisar la perspectiva y tendencia de la oferta y demanda educativa en dichos rangos, considerando, entre otros, los fenómenos que han terminado por orientar el debate desde el mercado, como la deserción, el perfil y la vocación, pero, igualmente, la necesidad de ubicar dicha triada en el escenario de la toma de decisiones que efectivamente debe rastrear este macro universo que no logra decantarse solo.

Tabla 1. Teorías sobre la deserción

\begin{tabular}{ll}
\hline $\begin{array}{c}\text { Teorías sobre la deserción (Tinto, 1987). } \\
\text { Supuestos básicos }\end{array}$ \\
\hline La conducta de los estudiantes refleja atributos propios y específicos \\
relacionados con las características psicológicas de cada individuo \\
(personalidad, disposición, motivación, habilidad y capacidad). \\
Es posible distinguir a los estudiantes que permanecen y a los desertores, \\
por los atributos de su personalidad que determinan diferentes respuestas a \\
circunstancias educativas similares.
\end{tabular}




\section{Teorías sobre la deserción (Tinto, 1987).}

Supuestos básicos

El éxito o el fracaso estudiantil es moldeado por las mismas fuerzas que configuran el éxito social en general y que definen el lugar que los individuos y las instituciones ocupan en la sociedad. Son elementos de predicción importantes del éxito escolar:

Sociales o ambientales

- El estatus social individual

- La raza

- El sexo

La deserción refleja el deseo intencional de las organizaciones educativas de restingir las oportunidades educativas y sociales a determinados grupos, aunque se declare lo contrario.

El estudiante contrasta los beneficios vinculados a la obtención de un determinado grado en una determinada institución, con los recursos financieros necesarios para hacer frente a la inversión que supone estudiar en la universidad.

El efecto del tamaño, la complejidad institucional, clos recursos disponibles, Organizacionales el ambiente y la existencia de estímulos diversos sobre la socialización de los estudiantes.

La conducta estudiantil es resultado de la interacción dinámica recíproca entre los ambientes y los individuos,

Fuente: Hincapié et al., 2012.

La expectativa planteada en la formación y oferta de programas en la educación superior colombiana está pasando del dato y las cifras a la revisión del quehacer académico estamental de las instituciones adscritas en dicho rango. No solo es el incremento de la oferta de programas en todos los niveles, sino el ascenso que tienen estos en diferentes campos, lo que debe cuestionar la formación ofrecida hasta ahora. De cierta manera, el elemento que viene dirigiendo dicho campo de análisis es el mercado, que con sobrados argumentos atiende el comportamiento del sector, pero no avanza en cuanto a las condiciones de cada disciplina y a la formación en el tiempo, ni resuelve cómo las instituciones de educación superior podrían atender dicho frente con sumo rigor.

Lograr que las empresas y la industria se involucren activamente en estas tareas. Trabajar con los gobiernos y el sector privado para aunar a los sectores empresariales y de la educación en la empresa común de llevar adelante sistemas de capacitación eficaces enclavados en la escuela y en las empresas. Esto supone identificar los asuntos polémicos que crean barreras y trabajar para echarlas abajo (Carlson, 2002, p. 140). 
La educación superior en Colombia necesita una revisión mucho más profunda que la que se hace teniendo en cuenta exclusivamente el comportamiento del mercado de la educación. La economía de la educación juega un papel trascendental para entender los argumentos y criterios sobre los cuales puede evaluarse el contexto en que se desenvuelve la formación superior, pregrado y posgrado, y que aún no hace parte de la gestión permanente de las instituciones de educación superior, cuyo marco de referencia continua en la trazabilidad del mercado, pero sin un trabajo amplio sobre lo que implica la oferta antes, durante y después de la formación, que finalmente, debe terminar siendo tutelada por las propias instituciones y no por las coincidencias ofrecidas de manera puntual.

Los orígenes de la Economía de la Educación y los diferentes enfoques se encuentran claramente desarrollados por Arjona, Tappata (h), Maradona y Sánchez. Los mismos afirman que, aunque ya en las ideas de Adam Smith y Alfred Marshall figuraba la educación como forma fundamental de inversión en capital humano, no fue sino hasta los '60 cuando la ciencia económica comenzó a formalizar modelos concretos acerca del impacto de la educación en la productividad, los ingresos laborales, y el crecimiento de los países (Calderón, et al., 2008, p. 21).

El estereotipo de mecanismo de flujo circular sobre el cual se ha extendido la relación de la formación académica superior, en tanto consecución de empleo u ocupación laboral, raya con la realidad, que debe evaluarse en cuanto a la estructura de valoración académica que debe darse en las instituciones de educación superior. La dificultad no está en la consecución de estudiantes ni en en la preferencia académica que finalmente escoja, ni en la deserción. La dificultad se encuentra en el marco de gestión de las instituciones en cuanto al seguimiento que deben establecer en relación con la deserción, el perfil y la vocación, el cual no puede limitarse al resultado, la graduación o la ocupación laboral.

La educación parecería ser el medio adecuado para tal fin. Sin embargo, es también la herramienta que permitiría perpetuar y acentuar las desigualdades. El resultado dependerá no sólo de los recursos que se destinen a la educación, y de los objetivos que los hacedores de políticas se planteen, sino también de la universalidad de la calidad en el sistema educativo y de las políticas sociales específicas que reduzcan los costos de oportunidad de los más pobres. Formichella y Londoño (2012, p. 89). 
Como herramienta analítica, el análisis multicriterio permite ordenar todos los frentes a los que haya lugar en virtud de la materialización y puesta de un problema en sí. No pretende despojar el problema o la situación considerada, sino la toma de principio a fin con el propósito de entenderla; su gran valía es que referencia todos los aspectos que le permitan atender eficazmente las razones sobre las que el problema o situación debe tratarse desde diferentes tópicos. En el momento en el que los argumentos desde diversas fuentes afloran, el problema pasa a ser tratado como un marco de trabajo para la toma de decisiones sobre criterios; en síntesis, el análisis multicriterio sólo pretende ubicar el tema en el centro de análisis y no por fuera.

Es importante mencionar que los análisis de los vínculos entre economía de la educación y el mercado laboral deben realizarse con base en estudios sobre egresados que verifiquen el perfil de los mismos y la pertinencia de los programas de educación superior, con respecto a la demanda del sector productivo, verificando la complementariedad de las teorías del capital humano, señalización del mercado y el concepto de satisfacción laboral, para explicar los sucesos acontecidos en los mercados laborales. Finalmente, es pertinente aclarar y sugerir, que se deben continuar realizando estudios sobre teorías de educación y mercado laboral; en aras de fortalecer la indefectible relación entre educación, rendimientos monetarios del trabajo y satisfacción laboral (Ángulo et al., 2012, p. 63).

La economía de la educación ofrece material importante sobre el cual aprehender con respecto al panorama real desde el que debe extenderse el marco de análisis y plancha de referencias desde el cual poder habilitar un trabajo permanente en materia de formación profesional de educación en pregrado y posgrado. En términos generales, cualquier profesión es válida, mientras cuente con la aprobación interinstitucional; no obstante, la educación aún no logra mostrar un panorama distinto al de la dependencia económica o realidad del contexto, que implica a que muchos de los factores que inciden en dicha decisión están por fuera del entorno de quien la emprende, y que, de paso, no cuenta con el acompañamiento en la lógica de la obtención de un producto.

Gran parte de la influencia que se tiene en el campo educativo radica en la necesidad de formar egresados cuyas características respondan a la demanda laboral vigente, lo cual conlleva a definir el estado actual de la práctica profesional y no el potencial. Ésta es una razón por la que los planes de estudio son instrumentos rígidos y cerrados, 
que consideran sólo lo establecido previamente, dejando a un lado la creatividad y el pensamiento innovador, tanto de profesores como de alumnos (Jiménez, 2002, p. 94).

El contexto en el que se desenvuelve la educación superior continúa recurriendo a la relación nominal de datos para advertir el comportamiento de la "economía o la gestión" de la educación superior. El margen de acción que puede entenderse en dicho frente termina siendo impulsado por campañas mediáticas, que como paliativos solo atienden el contexto en el que se desenvuelve la actividad, la economía o la administración del tema. La educación superior ha terminado por embarcarse en el escenario de los resultados, epicentro sobre el que las cifras son más reales que la trayectoria en el tiempo a partir de lo que puede suceder con los profesionales en ambas esferas de formación. Por el momento, la economía de la educación superior está por fuera de la realidad de las IES.

\section{Perspectiva de la educación superior en pregrado y posgrado en Colombia frente a la deserción profesional}

La educación superior, pregrado y posgrado, cuenta aún con un amplio prestigio entre la sociedad y la población interesada en formarse bajo la calidad profesional. No obstante, aún se considera que tras de ella existen aún diversos vacíos que no han podido tener respuesta clara frente a lo que implica la formación profesional de cara al deber y el liderazgo de las IES a la hora de responder transversalmente al tema. El trabajo relacionado con los elementos, parámetros y variables articulados a la toma de decisiones de formación aún es etéreo. El seguimiento transversal al quehacer y devenir de los profesionales se reduce a encuestas estadísticas sin alcance y la capacidad de las IES para orientar con respecto a la deserción, la orientación profesional y la vocación, que no es suficiente.

A diferencia de lo que ocurrió en otras latitudes del mundo, en donde los modelos de educación superior se reconstituyeron bajo pautas de orientación hacia la diferenciación institucional, en la región latinoamericana las tendencias y los cambios que se resintieron, hicieron referencia a un largo periodo de contracción de los recursos 
económicos, que provoco movimientos de adecuación constantes, que alteraron de manera definitiva la relación de participación y de conducción de los sectores tradicionales de la educación superior, los que deterioraron fuertemente la capacidad de legitimidad de los órganos de poder, de sus propósitos y de sus estrategias (Didriksson, 2008, p. 26).

El progreso institucional de la educación superior en Colombia ha estado acompañado del comportamiento económico que, por principio, continúa requiriendo personal formado en las IES para cubrir las demandas o vacantes laborales solicitadas por las empresas o el mercado laboral. A pesar de dicha relación, las IES aún no muestran progresos por fuera de sus instancias institucionales para tomar por cuenta propia los lineamientos que entre el mercado y la sociedad han terminado por definir la formación de educación para el futuro o por lo menos el que se requiere. En dicha perspectiva, el acceso a la educación superior prosigue en ascenso más no los análisis, estudios y pesquisas que dicho reporte debería arrojar a saber por cuenta de las propias IES.

Aunque éste trabajo no captura específicamente qué tipo de economías externas brinda la educación, y además el modelo únicamente tiene en cuenta los años de educación y no la calidad de está, por lo menos no subvalora la tasa de retorno social, ya que es muy bien sabido que si una sociedad tiene a sus miembros más educados, se puede beneficiar de distintas formas: incremento en el nivel de salud pública y por tanto menor costo de la misma, disminución de la tasa de fecundidad, mayor cultura, menor nivel de delincuencia que a la vez causa menor costo de mantener el orden público, menores niveles de desempleo, mayores niveles de calidad del empleo, entre otras (Marcillo \& Riascos, 2010, p. 18).

La transformación de la educación en todos sus frentes, pregrado y posgrado, ha terminado por habilitar una especie de avanzada por parte de las IES que, al paso de las exigencias, han confluido para resolver las demandas que el Ministerio de Educación Nacional (MEN) ha hecho y que, de manera estudiada y planteada, ha tenido importantes respuestas por cuenta de las primeras. En su defecto, la educación ha ampliado su capacidad académica al adherir a su quehacer actividades de extensión, internacionalización e investigación, triada con la que desafortunadamente no ha sido posible conjurar las exigencias que 
plantea la concepción de una formación amplia y profunda, y que efectivamente termine por colmar las aspiraciones de quienes logran encumbrar la profesión.

En el último cuarto de siglo se ha pasado de una Universidad de élites a la Universidad de masas. El crecimiento de casi todos los parámetros de los sistemas universitarios: número de estudiantes, número de centros, número de graduados, e t c., ha sido espectacular. También la financiación pública ha crecido, pero en menor proporción que los otros crecimientos y, últimamente, tiende a estancarse. Ahora que la demanda tradicional de enseñanza, la de los bachilleres, tiende a disminuir, nuevas demanda educación superior surgen.

En este panorama, que tiene aspectos positivos y negativos, las Universidades tendrán que diseñar estrategias de búsqueda y de gestión de recursos para la institución como un todo, combinando políticas de incentivo para los departamentos y profesores más competentes, con políticas de subsidio cruzado para la Universidad en su conjunto. Las Universidades, dentro de la multiplicidad de funciones que la caracterizan tendrán que examinar sus fortalezas y otear sus oportunidades y ajustar sus actividades en función de ellas y de sus recursos. No todos las Universidades podrán abordar con éxito todas las funciones y se producirá inevitablemente una diferenciación entre Universidades, circunstancia que en Europa empieza a producirse pero que en los Estados Unidos es moneda corriente desde hace mucho tiempo (Ortega, 2002, pp. 80-81).

El impulso obtenido por las IES conlleva precisamente a que estas asuman posición de timonel frente a lo que significa equilibrar la demanda y oferta educativa a escala superior. Si bien es un mercado de libre escogencia, en el que prevalecen diversos requerimientos, también es cierto que la oferta ha crecido de manera abismal. Sin embargo, el equilibrio no se refleja en la atomización de la demanda y la oferta, sino que se trata de entender la oferta educativa en el marco de una sábana interinstitucional en el que cada IES juega un papel crucial en lo que atañe a la decisión tomada por quien emprende el ciclo académico superior y desea continuarlo o decide allanar otras alternativas en otros frentes, no necesariamente correlacionados con la educación superior.

Ubicadas como instituciones de gran trascendencia para fines de desarrollo económico, cultural y social, las expectativas que se ciernen sobre las instituciones de educación 
superior y, en particular, las universidades están provocando presiones sin límites, en la redefinición de políticas y planes, la frecuencia de programas y alternativas en la búsqueda de nuevos modelos de organización. Estas tendencias e impactos hacen referencia a una dialéctica de escenarios que han alterado de forma significativa lo que la idea de universidad era hasta hace unas cuantas décadas, en relación a sus funciones, a sus sectores, a su gobernabilidad, a su calidad y a su lugar mismo en la sociedad. Habrá que destacar, no obstante, que el impacto del nuevo patrón social, tecnológico y productivo global ha propiciado la emergencia de redes, estructuras de cooperación y nuevos marcos de integración a nivel regional e interinstitucional que presentan, en tendencia, la posibilidad de construir un escenario alternativo o paralelo al de la competitividad institucionalizada y a la lógica del modelo (dominante) de mercado (Didriksson, 2008, p. 40).

El llamado a cambiar el modelo transmisor de la información y comunicador del conocimiento a uno dirigido a la creación y proyección del mismo le ha costado a las IES ponerlos al frente. Aún, el carácter académico-evaluativo protagoniza el margen de interacción entre los estudiantes de pregrado y posgrado, que a la par con las IES están involucrados en la disposición y otorgamiento de un título sin mayor avance en cuanto a lo que implica alternar dicha relación con los planteamientos del medio. El tinte de la relación, estimada en la definición de un contrato académico, no alcanza a indicar o mostrar los elementos, parámetros o variables con los cuales establecer el cubrimiento básico, mediano u óptimo de los máximos o mínimos del contrato.

En general, el resultado del balance que puede realizarse respecto a este giro de las políticas nacionales referidas a la educación superior, la ciencia y la tecnología en América Latina y el Caribe es que, sobre el supuesto que las empresas y las instituciones gubernamentales orientarían recursos frescos y se diversificarían las fuentes de financiamiento, se mantuvieron estancadas las inversiones hacia nuevos campos y hacia una posibilidad de nueva expansión de la demanda y la oferta; vaya, que se "dejo hacer" con políticas que navegaron en una suerte de indefinición, pero impulsando de forma directa orientaciones de mercado hacia la educación superior y la investigación con objetivos de corto plazo y de escasa trascendencia (Didriksson, 2008, p. 31). 
En el escenario internacional ha tomado fuerza la deserción escolar a escala de la formación superior, pregrado y posgrado, como una situación que viene adquiriendo trascendencia, en especial en los países emergentes o en vías de desarrollo. Sin embargo, es un fenómeno que ha adquirido relevancia en muchos otros países, precisamente por la desconexión entre las pretensiones académicas de las IES y los estudiantes a la par que con los requerimientos que la sociedad plantea con respecto a la oferta y demanda de profesionales. Si bien han sido combinados incentivos por el lado de la demanda y la oferta para que los estudiantes no terminen por fuera de la formación, la realidad es que el tema ha superado cualquier perspectiva integrada al comportamiento del mercado.

La deserción en los estudiantes es el resultado de la combinación y efecto de distintas variables. En éstas se encuentran características preuniversitarias, institucionales, familiares, individuales y las expectativas laborales. Estas influyen en la integración social y académica, las cuales a través del grado de motivación del estudiante provocan un efecto positivo -aumentando la probabilidad de permanecer- o negativo -presentando mayor probabilidad de desertar. El modelo conceptual de deserción/ permanencia propuesto permite proveer a administradores de la educación superior el marco para construir un plan de retención de estudiantes incorporando las necesidades individuales de sus estudiantes. Esto fue diseñado para que este modelo conceptual permita a administradores universitarios dedicar tiempo para realizar el seguimiento y evaluación permanente de las variables que afectan la integración social y académica, de manera de implementar estrategias.

Lo anterior, requiere recopilar de manera sistemática información sobre aspectos individuales, académicos, socioeconómicos de los estudiantes. Además, es posible profundizar los aspectos relacionados con las expectativas asociadas a autoeficacia y de desempeño y a los factores motivacionales relacionados con compromisos con metas académicas y de logro. Las expectativas y los factores motivacionales cambian durante su permanencia en la universidad. Por un lado, el seguimiento sistemático de éstos permitirá a los administradores universitarios orientar la gestión institucional a las necesidades de su propia población de estudiantes y, por otro, generar acciones de intervención que provoquen cambios en los factores de riesgo y así disminuir la deserción estudiantil (Díaz, 2008, pp. 82-83). 
La seguidilla de llamados al mejoramiento de la calidad o la certificación académica institucional es un indicador que atiende condiciones básicas de lo que implica estar en el proceso de formación en la educación superior. En dicho espacio, las IES deben extender de manera transversal el sentido de la certificación internacional obtenida. En la mayoría de los casos, la sociedad no alcanza a estimar el contenido de lo que implica la certificación de las IES, de allí que solo les interesa que esta pueda ofrecer carreras o profesiones con las cuales solventar sus necesidades puntuales. La certificación es una etapa como otras que deben surtir las IES, que, al margen de sus circunstancias, deben aflorar en distintos escenarios como el inherente a la relación con el estudiante-profesional.

Una primera observación que surge del seguimiento de desertores es que el problema quizá no sea tan grave como se presenta. En años recientes, documentos oficiales, particularmente el Programa Integral de Fortalecimiento Institucional, han sugerido una eficiencia terminal del 70\% como meta y han invitado a las carreras y universidades a someter propuestas al respecto. Pero quizá esta meta es poco realista y la fijación en el indicador puede llevar a medidas equivocadas. Según nuestros datos, casi la mitad de los desertores indica haberse trasladado a otra carrera o universidad. Abandonaron la carrera inicial, pero no el sistema. Si a estos jóvenes no les consideramos como desertores, los datos de deserción cambian de manera radical: si hay un $50 \%$ de deserción registrada por carrera, pero la mitad de ellos sigue en el sistema, la tasa real se ubicaría en un $25 \%$.

La deserción no resulta ser un asunto tan grave o complicado como a veces es presentado. Al mismo tiempo, el análisis de las razones que indican los desertores sugiere que hay medidas sencillas al alcance de la universidad para mejorar la atención al estudiante. El principal problema parece ser la decepción que siente el estudiante con la carrera en que se inscribió. Para atender este problema, quizá debemos empezar por dejar de rotular a los que cambian de opinión como desertores. Más bien, son decepcionados y habría que pensar en acciones que mejoran la atención al estudiante (Vries et al., 2011, pp. 46-47).

El mercado no solo puede entenderse como un asunto económico o de comportamiento de conductas, sino que atiende a patrones de ajustes y desequilibrios que terminan siendo atendidos por patrones institucionales y sociales, los cuales no alcanzan a fincarse en las 
encuestas o trabajos destinados a observar el comportamiento del mundo académico, antes, durante y después de lograr el título profesional. En términos generales, las IES se quedan con la información nominal y de registro relacionado con los estudiantes que iniciaron, desertaron y culminaron sus estudios a satisfacción. Al descontar cada una de ellas como etapas ajenas a un mismo contexto o al individualizarlas, el tema aún continúa postrado a lo poco que puedan hacer las IES y el MEN.

De lo anterior se percibe que el reto para hacer una gestión responsable de la educación superior es grande y se requieren seres humanos "humanos", con dones en los cuales los intereses colectivos primen. Coloquialmente, que se sitúen por encima del bien y del mal. Es necesario contar con herramientas que le permitan a las universidades o IES Ilevar a la práctica lo que se predica y evaluar el efecto de su gestión en la transformación social y el desarrollo, donde la RS es factor clave. Esto les facilitará el retorno a su papel protagónico en la construcción de la confianza que requiere Colombia, en las regiones y en su gente. Con el fin de responder a la sustentabilidad con pertinencia social se considera que la relación UniversidadEstado-Empresa debe incorporar alternativas que potencien una sólida relación de la Universidad con la sociedad civil, la contribución al bien público, la defensa de los valores civiles, la promoción y respaldo a los deberes ciudadanos y, en especial, la identificación con la comunidad local y, si es del caso, con la comunidad mundial ya que el logro de estos objetivos será el principal bastión en el tiempo (Hernández \& Acevedo, 2011, p. 171).

La desescolarización es uno entre muchos otros criterios que deben considerarse a la hora de entender el desempeño de la educación superior, pregrado y posgrado. La formación superior ha terminado por convertirse en otra alternativa más en la cadena de opciones que tienen los estudiantes a la hora de definir su futuro profesional. Es necesario aclarar que muchas de las actividades y funciones realizadas por las IES son desconocidas para los estudiantes, que solo apuestan por la formación como el único recurso de la transacción escolar que se crea entre ellos y las IES, criterio que ha tomado fuerza a pesar de los esfuerzos de estas por encontrar elementos de trabajo con los cuales abonar otras perspectivas en materia de formación para la educación superior. 
Los resultados encontrados en la presente investigación nos señalan la necesidad que tienen los jóvenes de contar con espacios educativos que les permitan conocer y desarrollar una visión a futuro, ya que aparte de ausencias en el conocimiento, los alumnos muestran falta de motivación, pues desconocen las fortalezas o debilidades que los lleve a la toma de decisiones. Sin lugar a duda, existen muchas causas que afectan la orientación; podemos mencionar entre ellas la no unificación de una metodología que permita uniformidad en la tarea, la falta de credibilidad en el servicio, ya que se le ve como una cuestión de trámite administrativo sin conocimiento de las teorías que justifican su importancia, la falta de profesionales con el perfil de orientadores o la falta de seriedad de las autoridades educativas en este proceso formativo, pues existen en el nivel medio, orientadores que sólo son vistos como prefectos.

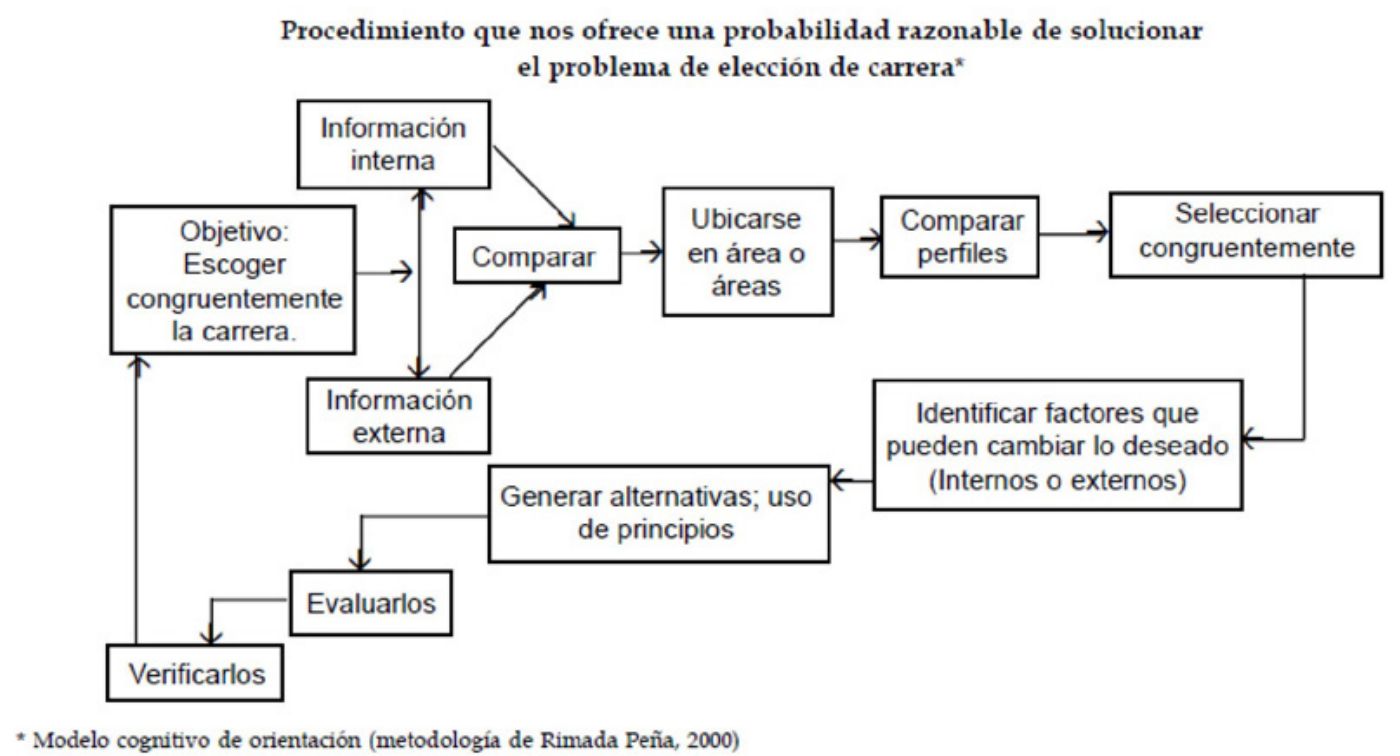

Figura 1. Modelo cognitivo de orientación

Fuente: De León y Rodríguez, 2008.

La disposición de argumentos, estudios o resultados que impliquen de manera robusta ejercicios encaminados a depurar la relación de la educación superior en diversos frentes solo ha contado con el interés de superar el tema de la deserción universitaria, dejando por fuera otros criterios igual o más importantes, como son la orientación, el perfil y la vocación profesional, cuyo recambio no está propiamente en la redención de las cifras a escala positiva, sino, por el contrario, sobre la manera como las IES atienden dicho fenómeno para que por encima del bajón de las cifras, el estudiante desertor pueda conforme 
a su paso por la IES acoger asignaturas, módulos o temas tras los cuales dicha deserción solo terminara por orientar su perfil sin perder realmente tiempo.

La evaluación de la calidad de las instituciones y el aseguramiento de la calidad de la educación superior son un requerimiento esencial de los países en la economía del conocimiento. El punto central que se propone en esta discusión es que, en la economía del conocimiento, la calidad de la educación superior y, principalmente, la educación universitaria, requiere evaluación permanente, sistemática e ininterrumpida. Efectivamente, la economía del conocimiento demanda niveles de calidad y excelencia en el accionar de la sociedad y, por ende, de las instituciones de educación superior (Ponce \& Palma, 2010, p. 12).

Es importante el rastreo hecho a través de encuestas, estudios o valoraciones para tratar de analizar los elementos a través de los cuales se pretende contextualizar el devenir de la educación superior. No obstante, es evidente que dicha trazabilidad está sujeta al grado de conexión transversal prevaleciente entre los diferentes actores-sujetos inscritos en la cadena. Igualmente, la formación pasa por una indagación profunda del estudiante o profesional, que debe evaluar el alcance de los estudios por los cuales ha optado de la mano con el acompañamiento y trascendencia real que las IES ofrecen a la hora de materializar, además del otorgamiento de títulos, el blindaje o tutela que deben tener de las ofertas académicas antes de ofertarse y posterior al logro del título profesional.

De modo general, se aprecia que mayor énfasis se da al proceso de admisión, es decir, al de inicio de la etapa formativa, y para ello las universidades cuentan con equipos muy completos de docentes y de profesionales especializados, que desarrollan estudios y mediciones sobre los resultados de los procesos de selección y sus características. No ocurre de igual modo con los procesos de inserción en el marcado laboral de los egresados. Trabajar en conjunto en ello, es una tarea que pendiente de las universidades (Becerra \& La Serna, 2010, pp. 61-62).

Es común que las IES en Colombia, y posiblemente en el mundo, pierden el rastro de los estudiantes-profesionales, aún de quienes hayan culminado "satisfactoriamente los estudios”. Es imperante para ellas que se extienda la capacidad de gestión institucional hacia elementos que no alcanzan a cubrirse con eventos o exaltaciones a los egresados. 
La labor en dicho periplo realmente es nueva para las IES, por cuanto aún aguardan de manera nominal que los estudiantes o los padres tomen la decisión de iniciar una carrera, más por convicción que por un claro conocimiento de lo que implica ello, y con el tiempo, la opción del grado profesional en cualquiera de los niveles, pregrado y/o posgrado es el punto de partida para dejar la relación sin reciprocidad.

Aunque el gobierno nacional abrió una convocatoria en el mes de mayo del 2010 para evitar la deserción universitaria, denominada Proyectos para el fomento de la permanencia estudiantil en educación superior (Universia, 2010), se debe tener en cuenta que la mayoría de las universidades adelantan programas de refuerzo académico y asistencia para mitigar la deserción universitaria y procurar por la permanencia de los estudiantes. Pero dichos esfuerzos y estudios están actualmente diseminados en distintas instituciones de todo el país, y aún no se cuenta con estudios confiables que abarcan la tipificación de las causas de deserción universitaria en general (Pinzón, 2011, p. 49).

El paso dado por la educación superior en materia de formación para el empleo o la disposición de mano de obra o profesionales especializados de mayor alto nivel no logra aún establecer el papel que debe fungir por cuenta de las IES, para que la educación superior, además de atractiva, cumpla en el tiempo con unos mínimos y máximos por quienes optan por dicha educación, pregrado y posgrados. En la actualidad, la educación superior enfrenta dificultades para establecer en un orden amplio, de contexto y profundo, lo que implica efectivamente el tomar una decisión que con el paso del tiempo puede tornarse angustiosa, molesta o poco relevante, si se trata de evaluar el papel cumplido por las instituciones de educación superior, por encima del acuerdo definido en el programa académico.

Concretamente, Enfermería, Económicas y Empresariales y las carreras de Ciencias (Matemáticas, Biología, etc.) son titulaciones que han resultado significativamente favorables para el ajuste educativo. En cuanto a la categoría ocupacional, tener un nivel educativo superior al requerido por el puesto (sobre educación) es más probable que ocurra si el empleo tiene lugar como trabajador no cualificado, mientras que los médicos MIR y los profesionales liberales aumentan la probabilidad de estar infra educados en su primer empleo. Por último, haciendo referencia a la movilidad laboral, observamos que: (i) la movilidad externa (interempresas) coadyuva al ajuste 
educativo, necesitando los graduados sobre educados pocos cambios de empleador para lograr el emparejamiento; (ii) la movilidad interna (intraempresa) «no permite» que los titulados logren el emparejamiento (Salas, 2004, pp. 276-277).

La relación planteada por la educación superior en materia de formación se ha caracterizado por mostrar a las instituciones encargadas de la misma de una manera un poco aletargada o pasiva en cuanto a lo que implica el seguimiento a la economía y/o el mercado de la educación superior. Su papel, definido en la disposición de programas para la consecución de un título profesional, constituye el claro reflejo de lo que implica para estas ubicarse en un punto de la relación. Aún, el precepto arraigado en el papel formal de la educación superior ha impedido que se atiendan otros frentes propios de la misma interacción, como el reconocimiento del proceso que se corre antes, durante y después del paso que un estudiante profesional debe incurrir para graduarse.

Entre la conciencia corporativista y el alto grado de competencia, negociaciones y alianzas que significa el proceso de socialización, se jugaría una manera de ser profesor en la universidad. Pero no la única, no olvidemos el aporte del individuo, de su transacción con esta realidad y la posibilidad de que, a partir de ahí, se encuentren otras salidas, otras propuestas. Según se ha visto en los casos estudiados, no es posible hablar de una única manera de ser profesional en la Universidad, y si bien hay cuestiones que se asemejan, que son comunes, hay otros en que no lo son, sutiles diferencias que muestran las modificaciones que pueden imprimirse en el desarrollo de la profesión y de la institución (Gewerc, 2001, p. 14).

El análisis difundido en cuanto a la estructura sobre la cual se comportan las instituciones de educación superior ampara la conducta funcional y operativa con las que estas actúan, en la medida en que la dirección de dicho proceso no solo corre por cuenta de ellas, sino del papel que cumplen, para el caso colombiano, instituciones como el Ministerio de Educación Nacional (MEN), el cual, dicho de paso, se ha preocupado más por la dependencia académica-universidad, que por seguir de principio a fin lo que implica para un individuo establecer los criterios que lo impulsan a tomar la decisión de elegir una carrera u optar por una formación de mayor raigambre, además de identificar a ciencia cierta los campos y el terreno que dicho profesional podría abarcar de la mano con las IES. 
Una educación por ciclos iría acompañada de un diseño curricular que permita al estudiante formarse en unas competencias generales y otras específicas de su profesión o disciplina y moverse dentro de su misma área del conocimiento y dialogar con otras áreas. Un currículo modular sería el modelo más adecuado para el diseño de los programas, pues, el estudiante puede combinar diversos esquemas de educación y trabajo, y de grados de presencialidad o educación a distancia. Cada uno de estos ciclos tendría la flexibilidad como principio curricular y académico de concepción y organización. La flexibilidad curricular se caracteriza por la posibilidad de responder creativa y rápidamente a los itinerarios intelectuales de estudiantes y profesores. Las unidades académicas posibiliten la apertura de nuevos temas, y dejen de lado el apego casi incuestionable al plan de estudios. Hay que estimular la construcción de espacios donde profesores y estudiantes tengan la libertad de asociarse para estudiar un tema de interés común (Celis \& Gómez, 2005, p. 12).

En las funciones sustantivas de las IES pueden estar aflorados los elementos que implican el habilitar puntos de trabajo con los estudiantes-profesionales que ingresan y egresan de ellas. En esencia, las actividades de mercadeo, foros académicos o vitrinas comerciales educativas son más consecuencia de una actividad que debe contenerse desde el interior de las IES. La economía de la educación es un elemento crucial para equilibrar lo hecho en cuanto a difusión institucional, pero para que este tenga eco, las IES deben modificar los esquemas y/o formatos como activan o interpretan la relación con los estudiantes. Aún se espera un poco más de cercanía o por lo menos tutoría cercana para ellos, para que temas como el mercado o la propia economía de la educación tenga efectivamente asiento.

\footnotetext{
Estas debilidades quizá pueden ser vistas más claramente si se analiza en los programas de posgrado, que son la base de la formación de científicos y técnicos. La matriculación en ellos es baja, en términos absolutos o relativos; la estructura de los programas de posgrado y de educación superior es, en general, dispareja en detrimento de las ciencias y de la ingeniería. Esto empuja a los estudiantes latinoamericanos, en posibilidad de hacerlo, a seguir sus estudios de posgrado en otros países. Mientras que en algunos países de América Latina existe lo que se podría llamar las bases mínimas para realizar actividades científicas y tecnológicas (infraestructura, investigadores, producción científica básica y aplicada, y programas de posgrado), éstas no son suficientes ni en calidad ni en cantidad (Moreno \& Ruiz, 2009, pp. 37-38).
} 
La búsqueda de formación profesional a escala tecnológica y profesional en los niveles de pregrado y posgrado aún esta sopesada en elementos que no son advertidos en los constantes estudios destinados a evaluar las condiciones de la demanda y oferta de educación posgradual. El análisis de la economía de la educación sólo llega a cumplir la escala de análisis estrictamente macroeconómico, por su correspondiente escenario de política estatal. A pesar de los esfuerzos encaminados a tener claridad sobre lo que implica estar en la economía de la formación en dichas escalas, los trabajos en dicho campo son pocos o por lo menos relativamente trascendentales a la hora de mediar entre lo que implica la orientación, el perfil y la vocación en la formación superior.

Castro y Levy describen lo que han denominado "la desprofesionalización de la educación superior". Debido a los grandes incrementos de matrícula en ese tramo, se generan más aspirantes a los puestos de trabajo que los que puede absorber el mercado, de modo que los graduados, al no poder encontrar una colocación acorde con sus estudios, aceptan cualquier empleo que puedan encontrar. Cuando los egresados de la educación superior no pueden encontrar trabajo concordante con sus estudios, sus títulos profesionales les ayudan a obtener un mejor empleo que los que no tienen estudios superiores (Carlson, 2002, p. 131).

En la mezcla de elementos de análisis y explicaciones, los aspectos que pueden servir para extender la sábana de trabajo sobre la que debe interpretarse la demanda y oferta académica de la educación superior. El reconocimiento a la labor de las IES pasa por el marco de gestión, que apunta a que estas puedan ser identificadas y estimadas en distintos frentes, aparte de la obtención de datos, cifras, estadísticas o referencias, sino teniendo en cuenta los elementos de trabajo que se requieren para entender el comportamiento de la educación superior, que pasan por la revisión que ellas mismas de manera interna hagan. Si bien las cifras pueden ser halagadoras o sanas, la realidad es que el esfuerzo de las IES no puede abocarse al quehacer del día, cuando la vocación es permanente.

Es decir, la tasa a la que los ingresos se elevan con los años de experiencia profesional es, en sí misma, una cuestión de elección individual. Desgraciadamente resulta imposible en la práctica separar los efectos de tales inversiones postescolares de la inversión normal en escolaridad formal, a menos que se suponga que todas las tasas 
de rendimiento de las inversiones escolares y postescolares se igualan en el margen (López \& Cárdenas, 2002, p. 103).

La deserción, la orientación, el perfil y la vocación profesional son elementos entrecruzados en una misma estela de posibilidades; así, más que un conato de crisis o llamado de atención, debe ser tomado bajo una constante reflexión. En dicho sentido, el papel que cumplen los estamentos encargados de la difusión de las IES es imperante, pero lo es mal aquel que tiene en cuenta a quienes conviven en tiempo real con aquellos que optan por iniciar su ciclo académico profesional. De esta manera, las instituciones deben entender que son múltiples los factores que inciden en cualquier decisión inherente al tema profesional; no obstante, son ellas quienes deben advertir a cuáles definitivamente le apuntan, para que los datos o cifras en ciertos tópicos sean tamizados desde la gestión más que de la administración.

\section{Diagrama 1. Clasificación de la deserción de acuerdo con el tiempo}

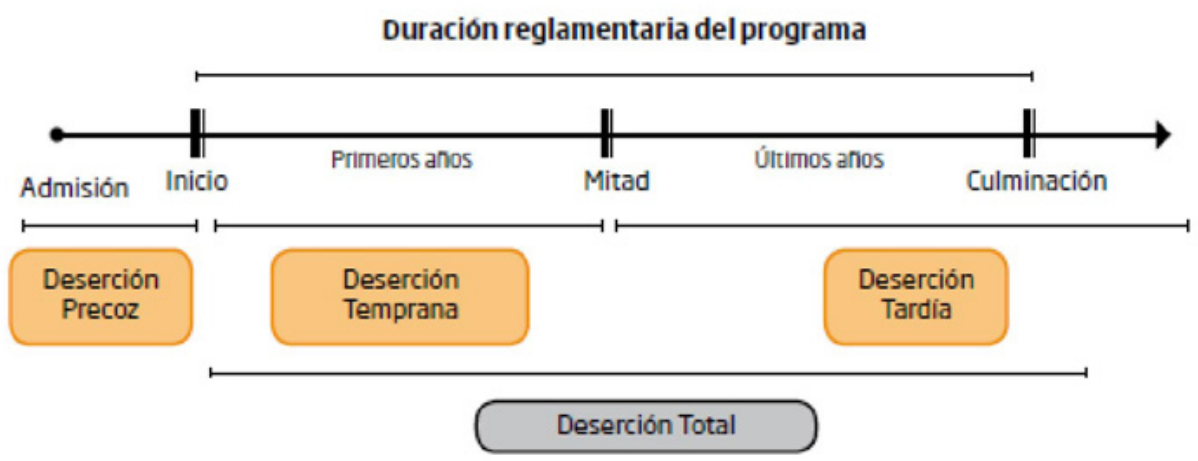

Figura 1. Clasificación de la deserción de acuerdo con el tiempo

Fuente: Ministerio de Educación Nacional, 2009.

Durante mucho tiempo, la oferta educativa de la educación superior en Colombia, al igual que en diversos países del mundo, estuvo caracterizada por ubicarse en el lado de la oferta. El número de profesionales en proporción al resto de la población era poco significativo. Al ubicarse la oferta de lado al lado de la demanda, la proporción de estudiantes en educación superior, pregrado y posgrado, ha venido acrecentándose de manera relevante, suceso que ha servido para connotar el papel jugado por la cobertura educativa en dicho frente académico. No obstante, la gestión organizacional destacada por las IES aparentemente prosigue en una relación pasiva con respecto a los cambios que presenta la economía y el mercado educativo. 


\section{La economía de la educación superior en la formación de pregrado y posgrado en Colombia}

La economía de la educación está a cargo de comprender el fenómeno educativo desde las instancias que hacen parte del mercado de la educación. No sólo se trata de la financiación de las IES o de los costos de la oferta académica de las distintas instituciones de educación superior, ni tampoco sobre la relación entre la carrera elegida y la remuneración sostenida por el mercado laboral. La economía de la educación expone la prospectiva de la educación como un todo, tanto para la demanda como para la oferta, al igual que de la sostenibilidad en el tiempo de la educación en todos sus niveles desde el mercado. Es precisamente este último cometido el centro de la economía de la educación, aun sin lograr.

La educación superior que involucra los tres actores importantes en esta industria: hogares, universidades y estudiantes. Se modela la demanda agregada de cupos por parte de hogares, la oferta agregada de cupos por parte de las universidades, así como la oferta universitaria de competencias profesionales, mientras que finalmente se modela la decisión óptima de esfuerzo por parte de estudiantes para culminar y graduarse de la universidad. En este contexto se determina la probabilidad de deserción que tiene un estudiante (Villa et al., 2013, p. 50).

El propósito de la economía de la educación tiende a tomar a cada uno de los actores inmiscuidos en dicha actividad a confrontarse o delimitarse frente a la trayectoria expuesta por los demás componentes de esta. En este sentido, la economía de la educación se encarga de dirimir las problemáticas que en materia de recursos deben considerar las IES para refrendar su actividad en el tiempo, a su vez, que la trascendencia que tienen las formaciones ofrecidas con cargo al costo de las profesionales y la esperanza matemática de la lógica remuneración equivalente que esperan los egresados, al igual que la sostenibilidad que en suma deben provocar los actores que como en cualquier otro escenario tienen la expectativa de que las condiciones mejoren en el largo plazo para todos como uno sólo. 
En este apartado se describe el enfoque económico convencional para el análisis de las decisiones educativas y se hace una aproximación teórica para comprender los economistas se han enfrentado al reto de analizar las decisiones educativas a lo largo de los últimos siglos. Pero los economistas no han sido los únicos en analizar la educación; este tema, ha sido analizado por diversas disciplinas entre las que se destacan la sociología, la demografía, la psicología y la economía (González, 2009, p. 8).

La preocupación por las finanzas en las IES tiende a ser el factor más relevante de trascendencia en primera instancia, centrada en una lógica de corto plazo, que no tiende a asegurar el mediano o largo plazo. La llamada competencia entre IES no existe, no solo porque la escogencia de esta recae en la familia, superando todo concepto de difusión o promoción, sino porque las IES deben trabajar conjuntamente, dado el principio de bien de servicio público al cual están adscritas, además de la impronta que deben acometer las agremiaciones de la educación superior, cuyo principal objetivo es destacar la educación colombiana, y de paso, cada una de las carreras que están bajo su autonomía, pero con autorización. En este sentido, las IES deben rescatar el carácter gremial de la educación superior nacional.

Algunos temas de la educación como la tasa de preferencia temporal (o de impaciencia) de los individuos, los gustos personales y la habilidad ha sido analizada por la psicología. Mientras la sociología se ha centrado en el papel del background social como determinante clave de las elecciones educativas. Por otra parte, la demografía ha hecho énfasis en la predicción del flujo y el stock de alumnos y graduados del sistema de educación. Finalmente, la economía desde una perspectiva ortodoxa empezó a estudiar el valor económico de la educación con la aparición de los economistas clásicos (González, 2009, p. 8).

En conjunto, las IES deben concentrarse en la oferta educativa del país, las apetencias académicas y profesionales, y los campos reales de acción que tienen no solo las profesiones escogidas, sino todo el orbe de la oferta educativa planteada por el sistema que aglutina a las IES, a través de asociaciones o grupos en las que estas se encuentran. La preocupación por las finanzas ha hecho a un lado la discusión sobre la demanda por carreras y/o las condiciones sobre las cuales en el tiempo accederían los futuros profesionales. Sin embargo, se continúa con la idea de que la oferta y la demanda educativa y laboral la resuelve el mercado, desconociendo que las IES también hacen parte del mercado, independiente de los regímenes, y que por tanto es allí donde deben concentrar el interés. 
Esta segmentación -dicotómica o tricotómica- del mercado laboral se convierte inevitablemente en un elemento de presión sobre el sistema educativo y sobre las políticas educativas. Más allá de la retórica sobre la igualdad de oportunidades y de las políticas impulsoras de una educación de calidad para todos, los sistemas educativos pueden seguir cumpliendo las funciones sociales discriminatorias que muchos les han atribuido e, incluso, estar encaminándose hacia nuevas formas, seguramente larvadas y sutiles, de segregación y de discriminación (Bajo, 2005, p. 669).

La suma de las IES, están en mora de tener una posición mucho más propositiva frente al futuro de la educación superior en Colombia y en gran parte del mundo. Si bien las agendas internacionales y los acuerdos entre países o convenios entre ellas proveen de cierto recurso de análisis, la realidad, es que el sistema educativo superior colombiano presenta una condición de diáspora, entre otras, por las distintas categorías y ofertas académicas dispares de iguales carreras, que más allá de la autonomía universitaria y de la exponente regulación, muestran que la educación en Colombia esta legislada, pero no reglada y mucho menos regulada, puesto que solo basta la aprobación de un registro para que distintas formas de educación superior ofrezcan la misma carrera o profesión.

La definición y la organización de determinado programa curricular en áreas disciplinares o profesionales se basan en un conjunto de criterios y supuestos sobre su racionalidad y utilidad futuras para los estudiantes que a él acceden. A su vez, la racionalidad y la utilidad se refieren a supuestos y expectativas sobre el futuro desempeño ocupacional del egresado. ¿En qué están basados estos supuestos y expectativas? ¿Cuál es la interpretación del contexto, tanto ocupacional como de desarrollo del conocimiento, que los sustenta? ¿Qué tan determinante es el peso de la tradición, de las inercias, de los intereses creados y del conocimiento del pasado? ¿Qué capacidad de interpretación de los cambios y tendencias del presente en el mundo del trabajo y del empleo, en los nuevos requerimientos de cualificación laboral y en las nuevas formas de organización, delimitación y producción de conocimientos, todos los cuales inciden significativamente sobre los criterios de organización curricular? ¿Cómo repercuten estos supuestos en la definición de las prácticas de enseñanza y evaluación, las cuales coadyuvan a generar un determinado perfil ocupacional? ¿Cómo permiten adoptar otras formas y escenarios de aprendizaje que favorezcan a un egresado su inserción en el mundo del trabajo? (Celis y Gómez, 2005, p. 1). 
En esencia, las IES tienen claro que el panorama en el largo plazo no está claro o por lo menos, está por fuera de lo que pueda apremiarse en un plan de desarrollo o plan estratégico para cada una de ellas. Hoy más que nunca se requiere tener claridad en cuanto a las pretensiones del gobierno colombiano para unificar o dilucidar un solo frente de acción en materia de educación superior, escenario para el que las alianzas, acuerdos, convenios, fusiones y redes deben ser contempladas, dado que el fenómeno internacional que caracteriza a este tipo de estamentos tiende a fomentar la unión entre ellas, más que la separación, cosa que no aplica en el caso colombiano, donde reina la división y poco trabajo articulado.

Un currículo modular sería el modelo más adecuado para el diseño de los programas, pues, el estudiante puede combinar diversos esquemas de educación y trabajo, y de grados de presencialidad o educación a distancia. Cada uno de estos ciclos tendría la flexibilidad como principio curricular y académico de concepción y organización. Las unidades académicas posibiliten la apertura de nuevos temas, y dejen de lado el apego casi incuestionable al plan de estudios (Celis y Gómez 2005, p. 12).

El universitario, a la par que el profesional, ha terminado por capturar de manera privada la autonomía universitaria que tanto defienden las IES ante el Estado. Finalmente, quien ingresa y egresa de estas, es quien ha terminado por hacer tanto vigilancia como comparaciones entre ellas, para sacar provecho de la ambigüedad de un rubro que no logro mediarse como asociaciones, gremios o mercado. Para ellos es clara la individualización que reportan las IES y que, contrario al matiz asociativo, corroboran de manera diáfana que son instituciones totalmente diferentes, y por lo tanto, de fácil captura o adquisición por parte de ellos, ya sea por costos, información, precios o servicios, parámetros en los que lo único que cabe es obtener el título sin importar en esencia ni la procedencia de las IES.

Desde un enfoque cualitativo, para evaluar un programa educativo es preciso conocer las características personales, profesionales y socioculturales de los maestros y los alumnos, analizar la estructura del programa, la pertinencia, los contenidos y las actividades de aprendizaje. Asimismo, se requiere evaluar la utilidad y adecuación de los recursos de aprendizaje desde los libros, las revistas, los videos y los discos interactivos, hasta las computadoras y sus programas y, por supuesto, Internet y sus inagotables fuentes de recursos (Perassi, 2008, pp. 9-10). 
Esta búsqueda hecha por los estudiantes y egresados es sobre la que deben replantearse las IES. La captura se basa en un reconocimiento pleno de lo que pueden ofrecer a los estudiantes, dejando claro lo que ellos pueden obtener de ellas. En este frente, cuando uno de los actores adquiere más información de las asociaciones, gremios o sectores, implica que hay fisuras en dicho campo que no son visibles para las IES. En este caso, se genera igualmente una relación agente-principal, en la que el oferente del recurso termina dominado por quien lo recibe, precisamente por manejo de información. El despliegue hecho por las IES para acercar más a los estudiantes o egresados, sin mayor respuesta, responde al caso típico de relación agente-principal.

La motivación del estudiante también puede tener fundamento en factores que atañen a la pertinencia educativa, en este caso basta recordar la importancia que tiene el nivel de satisfacción de las expectativas de futuro laboral y desarrollo personal del estudiante como fruto de su esfuerzo por mantenerse y culminar un programa académico de educación superior (MEN, 2009, p. 97).

El estudiante y el profesional esperan que lo prometido en el programa académico efectivamente sea real, dado que finalmente para ellos es el ejemplo claro de un contrato del cual se espera obtener los beneficios al momento de hacer cierre del proceso o culminar los estudios. Es por ello que entre las obligaciones entre las que se cuentan para las IES están las dirigidas a refrendar lo que en los programas o plegables académicos dan cuenta del quehacer, perfil y capacidad de desempeño profesional. Si bien las IES pueden contener dicho embate con la revisión del proceso académico llevado a cabo por cada individuo la realidad, es que en este momento las cuentas de haber están de lado de los estudiantes y egresados, y él debe se encuentra por cuenta de las IES y de los programas que ofrecen internamente.

En Colombia funcionan más de 16.000 programas en distintas áreas del conocimiento. Lo que no se sabe es en qué proporción los ciudadanos que están cursándolos sí están adquiriendo competencias útiles para nuestra sociedad. Editorial. Trabajo es lo que se espera (El Colombiano, 2010).

Se espera que en muy poco tiempo las IES expongan sus cuentas a la sociedad, por encima de las prerrogativas ministeriales, ofreciendo información sobre las carreras que 
tienen, el valor económico de las mismas en el tiempo, las remuneraciones efectivas de sus egresados, la probabilidad de continuidad, recambio o transformación de cada una de las profesiones ofrecidas y, la refrendación de dichos resultados por los estudiantes y egresados. De cierta manera, se está tejiendo el campo para que las IES entreguen un balance general de sus acciones, por encima de los articulados a sus respectivos órganos institucionales. En este sentido, se espera que las IES hagan rendición de cuentas a la sociedad como término de valor, mostrando así lo que representan para los lugares en donde se asientan.

Aquí se tratan dos enfoques, el del capital cultural por un lado y, por el otro, el del marketing educativo. Estos enfoques no son excluyentes, especialmente, bajo el nivel de perspectiva que se ha adelantado aquí (Bravo \& Mejía, 2010, p. 96).

En cuanto a la perdurabilidad y/o prospectiva de las IES, estas deben ir avanzado hacia la revisión de nuevos entornos estudiantiles, que superen la planeación por escenarios o la vinculación de tecnología a las aulas de clase. La planeación educativa ha dejado de ser un marco de referencia de programas, docentes y estudiantes; deben buscar la manera de hacer más comprensible el factor curricular del programa, el cual que debe ser un proceso consensuado y auspiciado por la institucionalidad. No es consecuente o claro para el estudiante que exista un programa mejor dotado que otro, contadas las proporciones. En este cometido, las IES deben pensarse como un todo y no solo desde los programas individuales.

\footnotetext{
Esta divergencia ha llevado a la OCDE a adoptar como política activa la mejora de las competencias de la fuerza de trabajo modificando los sistemas educativos. En Colombia, al igual que en el caso de la OCDE, se debe realizar una discusión sobre las competencias que favorecen nuestras instituciones educativas y las competencias que necesita el mercado (Mora, 2008, p.307).
}

La tarea está dirigida para que las IES sean vistas como un solo cuerpo a la hora de tomar la decisión de hacer parte de la agenda académica que estas expongan de manera unilateral o complementaria entre ellas. Lo que en el tiempo ofrecerán las IES estará sujeto a la agenda 
académica que pretenda llevar a cabo cada institución, ya sea en el orden ambiental, técnico o investigativo, para lo cual las hoy llamadas áreas sustantivas serán solo el soporte. En este sentido, más que programas o carreras profesionales, lo que delimitará el impacto y resultados de las IES estará trazado por apuestas concretas sobre las que se desplieguen esfuerzos, personal y recursos para hacer frente a dichas agendas que, finalmente, serán el factor diferencial entre las distintas IES que forman a los profesionales.

\section{Conclusiones}

La deserción ha dejado de connotar el problema de mayor interés para las IES, dado que, por lo general, la proporción de dicho suceso no es tan alta como se cree, dado que los estudiantes que desertan de una institución en muchos casos optan por terminar sus estudios en otra o en programas no formales. Aunque el seguimiento hecho a la materia tiene como base la individualización que se hace por parte de cada IES frente al mismo, la realidad es que este tema debe abordarse y corroborarse con todas las instituciones, haciendo seguimiento, precisamente, del cierre del proceso que hacen los estudiantes al desertar, que termina conciliado cuando es refrendado o aceptado en otra IES.

Es más amplio el contenido de información que tienen los estudiantes y egresados de las IES por encima de lo que estas consideran. No obstante, estas no se han preparado para considerar las nuevas exigencias que sobrevienen en cuanto a la manera de promover o proyectar la educación a futuro. Esta se encuentra enfocada en la flexibilidad en pleno sentido, y no solo en la programación amplia de horarios o mecanismos alternativos para acceder al conocimiento. Habrá estudiantes interesados en terminar en un tiempo inferior al estipulado o hacer carreras simultáneas, sin que esto implique retrasos, etc., atributos cuya respuesta ha sido más que tímida internamente en las IES y desatendida por completo por el MEN.

La captura pública-privada a la que están sometidas las IES implica para estas el gran reto, para la serie de balances, cuentas y rendiciones que tendrán que acometer en el tiempo. Para los grupos de interés o presión, que están al frente de dicha captura, es evidente que las IES deben hacer más de lo que se cree y deben comenzar a apropiarse 
y hacer frente a agendas de naturaleza nacional e internacional, que ponen a prueba la solidez académica que exponen. Es decir, que el futuro de las IES está sometido a la capacidad de hacer parte de las directrices internacionales que tienden a ubicar a estas como estamentos centrales para el futuro del planeta, y no solo espectadores ajenos a las realidades globales como los objetivos del milenio, el desarrollo sostenible y los protocolos internacionales, entre otros.

\section{Referencias bibliográficas}

(17 de abril de 2010). Trabajo es lo que se espera. El Colombiano, p. 5.

Ángulo, P., Grace, M., Quejada, R., \& Yánez, M. (2012). Educación, mercado de trabajo y satisfacción laboral: El problema de las teorías del capital humano y señalización de mercado. Revista de la Educación Superior. XLI(3), 51-66.

Bajo Santos, N. (2005). Educación, economía global y mercado laboral. Anuario Jurídico Económico. Escurialense. (38), 641-670.

Becerra, A. M., \& La Serna, K. (2010). Las competencias que demanda el mercado laboral de los profesionales del campo económico-empresarial en la actualidad. Documento de Discusión. Centro de Investigación de la Universidad del Pacífico.

Bondarenko Pisemskaya, N. (2007). Acerca de las definiciones de la calidad de la educación. Educere. 11(39), 613-621.

Bravo Castillo, M., \& Mejía Giraldo, A. (2010). Los retos de la educación superior en Colombia: Una reflexión sobre el fenómeno de la deserción universitaria. Asociación Colombiana de Facultades de Ingeniería (ACOFI), (10), 85-98.

Calderón, M.I., Ríos Olla, M. A., \& Ceccarini, M. F. (2008). Economía de la Educación (Primera edición). Argentina: Universidad Nacional del Cuyo.

Carlson, B. (2002). Educación y mercado de trabajo en América latina frente a la globalización. División de Desarrollo Productivo y Empresarial. Revista CEPAL 77, 123-141.

Castro, C., \& Levy, C. (2000): Myth, Reality and Reform: Higher Education Policy in Latin America. Washington, D.C.: Banco Interamericano de Desarrollo (BID).

Celis, J., \& Gómez, V. (2005). Factores de innovación curricular y académica en la educación superior. En: Revista ieRed: Revista Electrónica de la Red de Investigación Educativa [en línea], 1(2). 
Díaz, P.C. (2008). Modelo conceptual para la deserción estudiantil universitaria chilena. Estudios Pedagógicos, 34(2), 65-86.

De León, M.T., \& Rodríguez, M.R. (2008). El efecto de la orientación vocacional en la elección de carrera. Revista Mexicana de Orientación Educativa, 5(13), 11-16.

Del Valle García, O., \&Estrada, J. M. (2012). Nivel de satisfacción laboral de los egresados en la carrera de ingeniería Agronómica de la Universidad de Oriente. Núcleo Monagas.

Didriksson, A. (2008). Contexto global y regional de la educación superior en América Latina y el Caribe. En: A. L. Gazzola, \& A. Didriksson (eds.), Tendencias de la Educación Superior en América Latina y el Caribe (pp. 22-54). Caracas: IESALC-UNESCO.

Formichella, M.M., \& Londoño, S. (2012). Empleabilidad, educación y equidad social. Revista de Estudios Sociales, (47), 79-91.

Gewerc, A. (2001). Identidad profesional y trayectoria en la Universidad. Profesorado, revista de currículum y formación del profesorado, 5(2), 1-15.

Gómez Valencia, A. (19 de diciembre de 2010). Google sabe más de mí. Entrevista a Jesús Martin-Barbero. El colombiano, s.v. educación, p 1C.

Gómez Campo, V.M., \& Celis Giraldo, J.E. (2005). Factores de innovación curricular y académica en la educación superior. Revista Iberoamericana de Educación, 1l(2), 1-14.

Hernández P.R.D., \& Acevedo G. Augusto, C. (2011). Propuesta para la gestión responsable de la educación superior. Revista Soluciones de Postgrado EIA, (7), 153-173.

Hincapié, F. A., Hernández, A.W., Arteaga, J.S., Martínez, C.M., \& Pérez, R (2012). La deserción universitaria desde la perspectiva del estudiante. Informe de casos: programa microbiología y Bioanálisis. Revista Praxis, (8), 17-38.

Jiménez Ríos, E. (2002). La participación de los académicos en el diseño curricular de planes y programas de estudio en la UNAM. Perfiles Educativos. XXIV(96), 73-96.

Kemmerer, F. (1990). An Integrated Approach to Primary Teacher's Incentives”, Improving Educational Quality: A Global Perspective. En D. Chapman y C. Carrier (eds.), Nueva York: Greenwood Press.

Leyva López, S., \& Cárdenas Almagro, A. (2002). Economía de la educación: capital humano y rendimiento educativo. Análisis Económico. 17(36): 79-106.

Marcillo Y.E., \& Riascos, C. A. (2010). Una aproximación a los rendimientos sociales de la educación en Colombia y sus diferencias regionales. CIDSE, Universidad del Valle. Documentos de Trabajo no. 126. 1-25.

Mora, J. J. (2008). Sobre-educación en el mercado laboral colombiano, 10(19), 293-309. 
Moreno-Brid, J. C., \& Ruiz-Nápoles, P. (2009). La educación superior y el desarrollo económico en América Latina. Coordinación de Investigación. Sede Subregional de la C E P A L México. Serie Estudios y perspectivas 106.

Orozco Cruz, J.C., Olaya Toro, A., \& y Villate Duarte, V. (2009). ¿Calidad de la educación o educación de calidad? una preocupación más allá del mercado. Revista iberoamericana de educación, (51), 161-181.

Ortega, C.V. (2002). Tendencias de la Educación Universitaria en el Siglo XXI. Aáxx. Arbor CLXXIII(681), 67-81.

Perassi, Z. (2008). La evaluación en educación: un campo de controversias. - 1a ed. - San Luis: L.A.E. - Laboratorio de Alternativas Educativas. Recuperado de: http://lae.unsl.edu. ar/Ediciones/Libros_Electronicos.htm

Pinzón Cadena, L.L. (2011). Aplicando minería de datos al marketing educativo. Notas $D$ Marketing, 1(1), 45-61.

Ponce, E., \& Palma, Q. Á. (2010). Desafíos de la educación superior en la economía del conocimiento. Ingeniare, 18(1), 8-14.

Restrepo Posada, F. La responsabilidad de crear empresa y participar en el desarrollo del país también es asunto de los universitarios. Revista Universidad, Empresa y Estado, (1), 1-42.

Ricoachury, H., \& Lara, L. (2003). Los desertores. En: Cuadernos de Planeación. Bogotá: UPN. Bogotá. 1984.

Salas Velasco, M. (2004). La relación educación-economía: Un estudio del desajuste educativo de los titulados universitarios. Revista de la educación, (334), 259-278.

Villa Pérez, E., Misas Arango, M., Berrio Norman, M., \& Santacruz Rincón, S. (2013). Un modelo de educación superior y deserción universitaria: evidencia de la Pontificia Universidad Javeriana (Bogotá). Bogotá: Pontificia Universidad Javeriana - Facultad de Ciencias Económicas y Administrativas.

Vries de W, León A., P., Romero M., J. F., \& Hernández S, .I. (2011). ¿Desertores o decepcionados? Distintas causas para abandonar los estudios universitarios. Revista de la educación superior, $X L(4), 29-50$. 